\title{
Molecular Beam Measurement of the Hyperfine Structure of ${ }^{85} \mathbf{R b}^{19} \mathrm{~F}^{*}$
}

\author{
Jens C. Zorn, $\uparrow$ Thomas C. English, $\ddagger$ J. Thomas Dickinson, and David A. Stephenson $\S$ \\ Randall Laboratory of Physics, The University of Michigan, Ann Arbor, Michigan
}

(Received 11 July 1966)

\begin{abstract}
$$
\begin{aligned}
e q_{1} Q_{1} & =(-70.342 \pm 0.001) \mathrm{MHz}, \\
c_{1} & =(+0.52 \pm 0.02) \mathrm{kHz}, \\
c_{2} & =(+10.615 \pm 0.06) \mathrm{kHz}, \\
c_{3} & =(+0.80 \pm 0.06) \mathrm{kHz}, \\
c_{4} & =(+0.15 \pm 0.05) \mathrm{kHz} .
\end{aligned}
$$
\end{abstract}
Radio-frequency transitions between the hfs levels of the $J=1$ rotational state in ${ }^{85} \mathrm{Rb}^{19} \mathrm{~F}$ have been observed using a molecular beam electric resonance spectrometer. From these spectra we have obtained values for the constant $e q_{1} Q_{1}$, which characterizes the interaction between the electric quadrupole moment of the $\mathrm{Rb}$ nucleus with the molecular electric-field gradient; for the constants $c_{1}$ and $c_{2}$ which characterize the magnetic interaction between the $\mathrm{Rb}$ and $\mathrm{F}$ nuclei, respectively, and the rotational angular momentum $J$ of the molecule; and for the constants $c_{3}$ and $c_{4}$ which describe the magnetic coupling of the two nuclear spins. Measurements have been made on the first-five vibrational states. The results for $v=0$ are

Values of these constants for $v=1,2,3$, and 4 are given with somewhat less precision.

\section{INTRODUCTION}

$\mathbf{T}$ HE molecular beam electric resonance (MBER) method of spectroscopy is of great utility in the study of alkali halides because the spectrometer can select molecules of just one rotational state out of the many which are populated in a vaporized sample. The comparative simplicity of the spectra often makes it possible to isolate the spectral lines from each of the appreciably populated vibrational states, so the properties of molecules in known $(v, J)$ states can be studied in considerable detail. Transitions between the hyperfine sublevels of a single rotational state can be observed and measurements of these transitions, which occur at radio frequencies, give much information about the hyperfine interactions in the molecule. Our measurements ${ }^{2}$ of the radio-frequency spectrum of the $J=1, v=0,1,2,3$, and 4 states of ${ }^{85} \mathrm{Rb}^{19} \mathrm{~F}$ were motivated by our interests in nuclear hyperfine interactions and in vibrationally excited molecules; the results of these measurements are reported here.

The spectrum of RbF has been measured with molecular beam spectrometers using both electric ${ }^{3.4}$ and

* Research supported in part by the U. S. Atomic Energy Commission.

† Phoenix Faculty Fellow, 1965-1966.

$\ddagger$ NASA Fellow, 1963-1966; Present address: Physics Department, Harvard University, Cambridge, Mass.

$\S$ NSF Predoctoral Fellow.

iP. Kusch and V. W. Hughes, Encyclopedia of Physics (Springer-Verlag, Berlin, 1959), Vol. 37/I, pp. 46-50, 138-152. ${ }^{2} \mathrm{~A}$ preliminary report of this work has been given: $\mathrm{T}$. $\mathrm{C}$ English and J. C. Zorn, Bull. Am. Phys. Soc. 9, 661 (1964).

3 V. W. Hughes and L. Grabner, Phys. Rev. 79, 314 (1950).

${ }_{4}$ H. Lew, D. Morris, F, E. Geiger, and J. T. Eisinger, Can. J. Phys. 36, 171 (1958), magnetic ${ }^{5-7}$ resonance methods, and it has also been measured in a microwave absorption experiment. ${ }^{8}$ The first study of $\mathrm{RbF}$ in which it was possible to resolve the hyperfine structure completely, however, was done in the Yale molecular beam laboratory, where the measurement of most of the $J=1, v=0$ spectrum of ${ }^{85} \mathrm{Rb}^{19} \mathrm{~F}$ and ${ }^{87} \mathrm{Rb}^{19} \mathrm{~F}$ was carried through by Bonczyk. ${ }^{9}$ The present research is complementary to the work already done in that the hyperfine structure of the excited vibrational states has been measured with a precision comparable to that achieved for the ground state. The fact that our results for $v=0$ do not quite agree with those of Bonczyk and Hughes may prove to be significant if very detailed studies of the quadrupole hfs, as in a search for nuclear polarizability, ${ }^{10}$ are undertaken.

\section{MOLECULAR HAMILTONIAN AND EIGENVALUES}

The Hamiltonian operator of a diatomic, polar molecule in a ${ }^{1} \Sigma$ electronic state is taken to

${ }^{5}$ D. I. Bolef and H. J. Zeiger, Phys. Rev. 85, 799 (1952).

6 G. Bemski, W. A. Nierenberg, and H. B. Silsbee, Phys. Rev. $98,470(1955)$.

${ }^{7}$ F. Mehran, R. A. Brooks, and N. F. Ramsey, Phys. Rev. 141,93 (1966).

${ }^{8}$ S. E. Veazey and W. Gordy, Phys. Rev. 138, A1303 (1965).

${ }^{9}$ P. A. Bonczyk, Ph.D. dissertation, Yale University, 1965

(University Microfilms, Inc., Ann Arbor, Mich., 1966); P. A

Bonczyk and V. W. Hughes, Bull. Am. Phys. Soc. 10, 101 (1965).

${ }^{10}$ G. R. Gunther-Mohr, S. Geschwind, and C. H. Townes, Phys. Rev, 81, 289 (1951), 
be ${ }^{1,11}$

$$
\begin{aligned}
H=F_{v, J^{o p}}-e q_{1} Q_{1} \frac{3\left(\mathbf{I}_{1} \cdot \mathbf{J}\right)^{2}+\frac{3}{2}\left(\mathbf{I}_{1} \cdot \mathbf{J}\right)-\mathbf{I}_{1}^{2} \mathbf{J}^{2}}{2 I_{1}\left(2 I_{1}-1\right)(2 J-1)(2 J+3)}+c_{\mathbf{1}} \mathbf{I}_{1} \cdot \mathbf{J}+c_{2} \mathbf{I}_{2} \cdot \mathbf{J} \\
\quad+c_{3} \frac{3\left(\mathbf{I}_{1} \cdot \mathbf{J}\right)\left(\mathbf{I}_{2} \cdot \mathbf{J}\right)+3\left(\mathbf{I}_{2} \cdot \mathbf{J}\right)\left(\mathbf{I}_{1} \cdot \mathbf{J}\right)-2\left(\mathbf{I}_{1} \cdot \mathbf{I}_{2}\right) J(J+1)}{(2 J-1)(2 J+3)}+c_{4} \mathbf{I}_{1} \cdot \mathbf{I}_{\mathbf{2}}-\boldsymbol{u} \cdot \mathbf{E} .
\end{aligned}
$$

$F_{v J^{\text {op }}}$ encompasses the vibrational and rotational energies of the molecule in the electronic ground state, and, since we are examining transitions between hyperfine levels, it is not necessary to discuss this first term further. The second term of the Hamiltonian represents the interaction of the rubidium's nuclear electric quadrupole moment $Q_{1}$ with the molecule's electricfield gradient $q_{1} . \mathbf{I}_{1}$ and $\mathbf{I}_{2}$ are the spin operators of the $\mathrm{Rb}$ and $\mathrm{F}$ nuclei, respectively. Since the fluorine nucleus has a spin of one-half, it has no observable quadrupole coupling. The third and fourth terms of Eq. (1) represent the interaction between the magnetic moments of each nucleus and the magnetic field which arises from the molecule's rotational angular momentum $\mathrm{J}$. The fifth and sixth terms of the Hamiltonian represent the magnetic (spin-spin) interactions ${ }^{12}$ of the two nuclei with each other. The last term in the Hamiltonian represents the interaction of the molecule's permanent electric dipole moment $\boldsymbol{u}$ with an externally applied electric field E. To be more general one should also include coupling to the external magnetic field, but the magnetic fields in the resonance region of the present experiment were kept small enough that Zeeman effects ${ }^{13}$ were not visible in the spectrum.

The energy eigenvalues of the Hamiltonian under conditions when coupling to external fields is very small (the very-weak-field case, where the appropriate representation is $\left.\left|I_{1}, I_{2}, J, F_{1}, F, M_{F}\right\rangle\right)$ are well known..$^{14,15}$ As emphasized by Bonczyk ${ }^{9}$, the offdiagonal contributions of the quadrupole coupling ${ }^{15}$ must be included if the observed spectra are to be satisfactorily described.

The differences between the molecular energy levels, as found from the Hamiltonian, can be divided by the Planck constant to give the frequencies of the spectral lines which are expected under very-weak-field conditions. We express the frequency of the $k$ th line as

$$
f_{k}=\sum_{i=0}^{4} c_{i} G_{i k}\left(I_{1}, I_{2}, J, F_{1}, F\right)
$$

where the $c_{i}$ are the coupling constants characteristic of

\footnotetext{
${ }^{11}$ G. Gräff, W. Paul, and C. Schlier, Z. Physik 153, 38 (1958).

${ }_{12}$ C. Schlier, Fortschr. Physik 9, 455 (1961).

${ }^{13}$ The combined Stark-Zeeman spectrum of $\mathrm{RbF}$ has been studied by G. Gräff, R. Schönwasser, and M. Tonutti, Proc. Symp. Mol. Struct. Spectry., Ohio State Univ., 1966, 64 (1966); Z. Physik (to be published).

${ }_{14}$ V. W. Hughes and L. Grabner, Phys. Rev. 79, 829 (1950).

16 J. Bardeen and C. H. Townes, Phys. Rev. 73, 627, 1204 (1948); C. H. Townes and A. L. Schawlow, Microreve Spectroscopy (McGraw-Hill Book Co., Inc., New York, 1955), pp. 155-164, 517-521.
}

the particular molecule (for convenience in notation here and in Sec. IV, $c_{0}$ represents the quadrupole interaction including the contributions which are not diagonal in $J$ ), and the $G_{i k}$ are known ${ }^{1}$ functions of the angular momenta of the molecule. For ${ }^{85} \mathrm{Rb}^{19} \mathrm{~F}$ in the $J=1$ state there are 11 transitions between quadrupole levels which are allowed by the selection rules ${ }^{14}$ which hold in very weak fields; we label these transitions by their initial and final values of $F_{1}, F$ as, for example, $\left(\frac{3}{2}, 2\right)-\left(\frac{7}{2}, 4\right)$. Transitions of this type are indicated on the diagram of the $J=1$ hyperfine structure shown in Fig. 1. If $n_{v}$ of these transitions are observed for a given vibrational state $v$, then Eq. (2) above represents $n_{v}$ simultaneous equations in the five unknowns $c_{i}$.

\section{EXPERIMENT: APPARATUS}

The molecular beam spectrometer used for these studies is similar to others described in the earlier literature. ${ }^{1,16}$ The apparatus is described in full detail in our reports ${ }^{17}$ on the hyperfine-structure measurements on CsF. Only a few important features and dimensions of the spectrometer are given here.

The molecular beam issues from a 0.025 -cm-diam hole in the front of the source oven. This oven is constructed of nickel or of boron nitride in a conventional form $^{1}$; it is electrically heated to a typical operating temperature of $1050^{\circ} \mathrm{K}$. The detector, $1.62 \mathrm{~m}$ from the oven, uses a hot tungsten-wire surface ionizer; the detector has an effective width of $0.02 \mathrm{~cm}$ and a height of $0.10 \mathrm{~cm}$. The minute current from the detected beam is measured with a vibrating-reed electrometer whose output is digitized and recorded for subsequent analysis.

Electric four-pole fields provide the state selection. ${ }^{18}$ When the apparatus is aligned properly with all stops in place, a signal from state selected molecules of about $8 \times 10^{-13} \mathrm{~A}$ on a background of about $2 \times 10^{-13} \mathrm{~A}$ is obtained. Transitions are measured by alternately applying and removing the rf signal and noting the correlated change in detector current. The most intense transitions result in detector current changes of about $4 \times 10^{-14} \mathrm{~A}$. The raw signal-to-noise ratio for these large transitions is about 10:1 when the detector time

${ }^{16}$ C. Schlier, Z. Physik 147, 600 (1957).

${ }^{17} \mathrm{~T}$. C. English, Ph.D. dissertation, University of Michigan, 1966 (University Microfilms, Ann Arbor, Mich., 1966); T. C. English and J. C. Zorn (to be published).

${ }_{18}$ H. Bennewitz, W. Paul, and C. Schlier, Z. Physik 141, 6 (1955). 
constant is $1 \mathrm{sec}$, and averaging the numerical results of several passes over the spectral line increases the effective signal-to-noise ratio by a factor of 3 or so.

It is of some interest to note that small fluctuations in the vacuum appear to be one of the major sources of noise in the experiment reported here; ion background from the detector wire gave relatively little trouble. When the spectrometer's operation is noisy, we generally observe that the main chamber vacuum of $\sim 10^{-7}$ torr is fluctuating by $\widetilde{>} 3 \times 10^{-9}$ torr; these fluctuations occur as bursts toward higher pressure and they are inevitably accompanied by a corresponding, sharp change in the detected beam current. The apparent duration of these pressure bursts is less than $1 \mathrm{sec}$; how much less we cannot say because the time constants of the vacuum gauges and beam detector are too long. The origin of these vacuum bursts is uncertain; some may arise from the sudden release of trapped pockets of gas, as from the lubricated surface of an O-ring seal,,$^{19}$ and some bursts can be correlated with boiling noises in the diffusion pumps. We have been able to improve our system's operation considerably by using diffusion pumps which we have individually selected and tested for quiet vacuum production; the NRC HK-4-750 diffusion pumps ${ }^{20}$ with DowCorning DC-705 oil have proved somewhat more satisfactory than others we have tested.

The $C$ field, ${ }^{21}$ in which the transitions are induced, permits the radio-frequency signal to be applied over a $51-\mathrm{cm}$-long portion of the beam path. Since the molecular velocities are about $5 \times 10^{4} \mathrm{~cm} / \mathrm{sec}$, the transit time through the resonance region is about $1 \mathrm{msec}$ : This implies ${ }^{22}$ that the spectral linewidth should be $1 \mathrm{kHz}$, and this is about what is observed (see Fig. 2) . The $C$-field electrodes are spaced $1 \mathrm{~cm}$ apart and a $1.5-\mathrm{V}$-dc potential difference is maintained across them; the electric field is not highly homogeneous, but

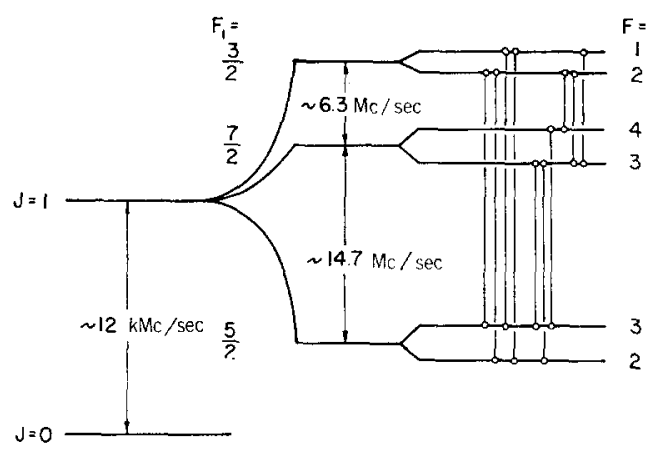

Fig. 1. Energy levels of the $J=1$ rotational state of ${ }^{85} \mathrm{Rb}{ }^{19} \mathrm{~F}$ with no external field applied. Observed transitions are indicated.

${ }^{19}$ U. R. Bance and E. H. Harden, Vacuum 15, 437 (1965).

${ }^{20}$ Manufactured by the National Research Corporation, Newton Highiands, Mass.

${ }^{21}$ J. W. Trischka, Phys. Rev. 74, 718 (1948); D. T. F. Marple

and J. W. Trischka, ibid. 103, 597 (1956).

${ }_{22}$ N. F. Ramsey, Molecular Beams (Oxford University Press, London, 1956), pp. 118-124.

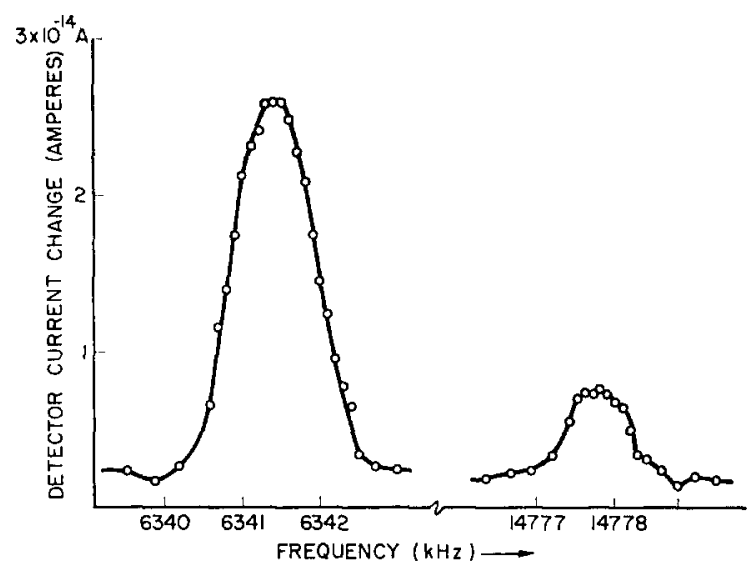

FIG. 2. Observed signals for the $\left(\frac{3}{2}, 1\right)-\left(\frac{7}{2}, 3\right)$ transition at $(6341.45 \pm 0.15) \mathrm{kHz}$ and for the $\left(\frac{7}{2}, 4\right)-\left(\frac{5}{2}, 3\right)$ transition at $(14777.85 \pm 0.25) \mathrm{kHz}$. The dc field strength is $1.5 \mathrm{~V} / \mathrm{cm}$.

the contributions to linewidth from dc field inhomogeneity are negligible at these very weak fields. The magnetic field over the entire transition region is reduced to less than $50 \mathrm{mG}$ by minimizing the magnetic materials in the apparatus, and by cancelling the earth's field with a set of coils.

The radio-frequency signal, derived from a synthesizer system from which the frequencies are known to one part in $10^{7}$ or better, is applied to the $C$ field in such a way that the rf field has a large component perpendicular to the dc field over the cross section of the beam. The rf Stark effect is hard to estimate, but the spectral lines used to determine the constants of the RbF molecule are all taken at a level of rf and dc below the point where the line shape or position are observed (to within limits imposed by noise) to depend significantly on the size of the external fields.

\section{EXPERIMENT: RESULTS AND DISCUSSION}

The four-pole, electric state selectors ${ }^{18}$ have a focusing property for those molecules of the $J=1$ rotational state which have $M_{J}=0$ in high fields. In order to be observable in our apparatus, the transitions must occur between those very-weak-field hfs states which correspond to different values of $\left|M_{J}\right|$ in the strong fields of the state selectors. Moreover, the $\Delta F_{1}=1,2$ transitions between quadrupole levels are governed by the selection rules ${ }^{14} \Delta F=0,1,2 ; \Delta M_{F}=0,1$. For each vibrational state, a total of nine transitions out of the $F_{1}=\frac{3}{2}$ and $F_{1}=\frac{7}{2}$ sublevels both obey the selection rules and satisfy the observability criterion. In addition, two transitions, $\left(\frac{7}{2}, 3\right)-\left(\frac{5}{2}, 2\right)$ and $\left(\frac{7}{2}, 3\right)-\left(\frac{5}{2}, 3\right)$, which occur between initial and final states which both correspond to $\left|M_{J}\right|=1$ in high field, are observed; the signals from these lines are quite low and we assume that their observation arises from nonadiabatic transitions, ${ }^{23}$ probably in the regions between the state

${ }^{23}$ L. Grabner and V. W. Hughes, Phys. Rev. 79, 819 (1950). 
TaBlE I. Hyperfine-structure transition frequencies in the $J=1$ state of ${ }^{85} \mathrm{Rb}^{19} \mathrm{~F}$.

\begin{tabular}{|c|c|c|c|c|c|c|}
\hline $\begin{array}{l}\text { Transition } \\
\left(F_{1}, F\right)-\left(F_{1}^{\prime}, F^{\prime}\right)\end{array}$ & $I^{\mathbf{b}}$ & $\nu=0$ & $v=1$ & $v=2$ & $v=3$ & $v=4$ \\
\hline $\begin{array}{l}\left(\frac{3}{2}, 2\right)-\left(\frac{7}{2}, 4\right) \\
\left(\frac{3}{2}, 2\right)-\left(\frac{7}{2}, 3\right)\end{array}$ & $\begin{array}{r}40 \\
4\end{array}$ & $\begin{array}{l}6320.9 \pm 0.15 \\
6334.5 \pm 0.25\end{array}$ & $6250.20 \pm 0.15$ & $6180.38 \pm 0.15$ & $6111.55 \pm 0.20$ & $6043.60 \pm 0.35$ \\
\hline$\left(\frac{3}{2}, 1\right)-\left(\frac{7}{2}, 3\right)$ & 28 & $6341.45 \pm 0.15$ & $6270.55 \pm 0.15$ & $6200.6 \pm 0.20$ & $6131.40 \pm 0.20$ & $6063.35 \pm 0.40$ \\
\hline $\begin{array}{l}\left(\frac{7}{2}, 3\right)-\left(\frac{5}{2}, 3\right) \\
\left(\frac{7}{2}, 3\right)-\left(\frac{5}{2}, 2\right) \\
\left(\frac{7}{2}, 4\right)-\left(\frac{5}{2}, 3\right)\end{array}$ & $\begin{array}{l}0.8 \\
2 \\
6\end{array}$ & $\begin{array}{ll}14 & 764.10 \pm 0.25 \\
14 & 766.35 \pm 0.25 \\
14 & 777.85 \pm 0.25\end{array}$ & & & & \\
\hline $\begin{array}{l}\left(\frac{3}{2}, 2\right)-\left(\frac{5}{2}, 3\right) \\
\left(\frac{3}{2}, 2\right)-\left(\frac{5}{2}, 2\right) \\
\left(\frac{3}{2}, 1\right)-\left(\frac{5}{2}, 3\right) \\
\left(\frac{3}{2}, 1\right)-\left(\frac{5}{2}, 2\right)\end{array}$ & $\begin{array}{l}24 \\
12 \\
10 \\
18\end{array}$ & $\begin{array}{ll}21 & 099.10 \pm 0.15 \\
21 & 100.85 \pm 0.15 \\
21 & 106.20 \pm 0.15 \\
21 & 108.30 \pm 0.15\end{array}$ & $\begin{array}{ll}20 & 863.10 \pm 0.15 \\
20 & 864.70 \pm 0.15 \\
20 & 870.25 \pm 0.15 \\
20 & 872.27 \pm 0.15\end{array}$ & $\begin{array}{ll}20 & 630.20 \pm 0.15 \\
20 & 631.80 \pm 0.20 \\
20 & 637.25 \pm 0.15 \\
20 & 639.32 \pm 0.15\end{array}$ & $\begin{array}{l}\left\{\begin{array}{l}20401 \pm 1 \\
\text { Unresolved pair }\end{array}\right. \\
20407.25 \pm 0.2 \\
20409.35 \pm 0.3\end{array}$ & $\begin{array}{l} \begin{cases}20 & 174.5 \pm 1 \\
\text { Unresolved pair }\end{cases} \\
\begin{cases}20 & 183 \pm 2 \\
\text { Unresolved pair }\end{cases} \end{array}$ \\
\hline
\end{tabular}

a Frequencies are given in kiloherz. The uncertainties given in this table arise from the difficulty in assigning a central frequency to an observed line; they do not include the possible systematic error from rf Stark shifts and other effects discussed in Sec. IV.

selectors and the $C$ field, along with the action of the $\mathrm{rf}$; these lines are given less weight in the analysis of data than the others observed in $v=0$.

The frequencies observed for the various hyperfinestructure transitions in ${ }^{85} \mathrm{Rb}^{19} \mathrm{~F}, f_{k}$ obs, are given in Table I; the earlier work ${ }^{3,24}$ done on $\mathrm{RbF}$ enables one to assign initial and final states with little difficulty. It remains to select a set of constants for each vibrational state so that the frequencies $f_{k}$ of $\mathrm{Eq}$. (2) agree as closely as possible with the observations. We write:

$$
\begin{aligned}
f_{k} \text { obs }-\sum_{i=0}^{4} c_{2} G_{i k}\left(I_{1}, I_{2}, J, F_{1}, F, M_{F}\right) & =r_{k} ; \\
k & =1,2, \cdots, n_{v},
\end{aligned}
$$

and choose that set of molecular constants $c_{i}$ which minimizes the weighted sum of squares $\sum_{k} w_{k} r_{k}^{2}$. This is done conveniently on an IBM 7090 computer ${ }^{25}$ Results for the vibrational states $v=0,1,2,3$, and 4 are given in Table II.

For $v=0$, the 10 measured spectral line frequencies are weighted in a manner which takes into account the uncertainty in apparent central frequency, reproducibility of shape, and a subjective estimate of line quality. Fairly drastic changes in the weighting will cause the output value of the $v=0$ constants to vary somewhat, but the range of the variation is generally inside the quoted uncertainty. If all lines are weighted equally, for example, then the absolute value of the quadrupole coupling constant is somewhat lower $(-70.34160 \mathrm{MHz})$ and the value of $c_{2}$ is just a bit higher $(10.668 \mathrm{kHz})$ than those obtained from the weighted lines, while the values of $c_{1}, c_{8}$, and $c_{4}$ are essentially unchanged.

${ }^{24}$ J. C. Zorn, P. A. Bonczyk, and V. W. Hughes, Bull. Am. Phys. Soc. 7, 44 (1962).

${ }_{25}$ We are grateful to $\vec{F}$. Stephenson for assistance with machine computations. b The column labeled $I$ gives the approximate signal, in units of $10^{-16} \mathrm{~A}$ which is observed from each of the $v=0$ transitions. The intensities from the higher vibrational states were less, as expected from the reduced population of these states. The $\left(\frac{7}{2}, 4\right)-\left(\frac{5}{2}, 2\right)$ transition was not intense enough to be measured.

For the higher vibrational states $(v=1,2,3,4)$, the lines corresponding to those of lesser weight in $v=0$ were not strong enough to be measured [except the $\left(\frac{3}{2}, 2\right)-\left(\frac{5}{2}, 2\right)$ transition which was seen in $v=1$ and $v=2]$, so all the observed lines were used with equal weight to compute the hfs constants for $v>0$. Because all lines were not seen, the precision of these hfs constants, particularly $c_{1}, c_{3}$, and $c_{4}$, is not as high as that quoted for $v=0$.

It is seen from the data that the electric-quadrupole interaction does not have a purely linear dependence on the vibrational quantum state; moreover it has been shown ${ }^{5}$ that the $\mathrm{RbF}$ quadrupole interaction (albeit in ${ }^{87} \mathrm{Rb}^{19} \mathrm{~F}$ ) changes with rotational state by an amount corresponding to a value of $26 \pm 4 \mathrm{~Hz}$ for the coefficient $e Q q^{(J)}$ in $\mathrm{Eq}$. (4) below. It is reasonable to assume that the value of $e Q q^{(J)}$ for ${ }^{85} \mathrm{Rb}^{19} \mathrm{~F}$ is on the order of $50 \mathrm{~Hz}$, but we cannot measure the coefficient with the present apparatus. These variations in the quadrupole interaction are assumed to be from changes in the value of the electric-field gradient $q$, so we write

$$
\begin{aligned}
e Q q(v, J)= & e Q\left[q^{(0)}+q^{(v)}\left(v+\frac{1}{2}\right)+q^{(2 v)}\left(v+\frac{1}{2}\right)^{2}+\cdots\right. \\
& \left.+q^{(J)} J(J+1)+q^{(2 J)} J^{2}(J+1)^{2}+\cdots\right],
\end{aligned}
$$

where the quantities $q^{(v)}, q^{(J)}$, etc. are calculated directly from the spectra. For ${ }^{85} \mathrm{Rb}^{19} \mathrm{~F}$ in the $J=1$ state we find

$$
\begin{aligned}
& e Q q^{(0)}=-70.739 \mathrm{MHz}, \\
& e Q q^{(v)}=+0.797 \mathrm{MHz}, \\
& e Q q^{(2 v)}=-0.005 \mathrm{MHz} .
\end{aligned}
$$

Theories of molecular structure and their predictions for values of various constants of the molecule, however, usually have the internuclear separation $R$ as an independent variable, so it is most useful if the values of 
TABLE II. Hyperfine coupling constants for the $J=1$ state of ${ }^{85} \mathrm{Rb}^{19} \mathrm{~F}$.

\begin{tabular}{|c|c|c|c|c|c|}
\hline & $e q_{1} Q_{1}(\mathrm{MHz})$ & $c_{1}(\mathrm{kHz})$ & $c_{2}(\mathrm{kHz})$ & $c_{3}(\mathrm{kHz})$ & $c_{4}(\mathrm{kHz})$ \\
\hline \multicolumn{6}{|c|}{ Present experiment } \\
\hline$y=0$ & $(-70.342 \pm 0.001)$ & $(+0.52 \pm 0.02)$ & $(+10.615 \pm 0.06)$ & $(+0.80 \pm 0.06)$ & $(+0.15 \pm 0.05)$ \\
\hline$v=1$ & $(-69.555 \pm 0.002)$ & $(+0.52 \pm 0.05)$ & $(+10.483 \pm 0.10)$ & $(+0.77 \pm 0.10)$ & $(+0.06 \pm 0.12)$ \\
\hline$v=2$ & $(-68.779 \pm 0.002)$ & $(+0.52 \pm 0.08)$ & $(+10.406 \pm 0.10)$ & $(+0.76 \pm 0.10)$ & $+(0.07 \pm 0.15)$ \\
\hline$v=3$ & $(-68.013 \pm 0.003)$ & $(+0.52 \pm 0.15)$ & $(+10.205 \pm 0.15)$ & $(+0.77 \pm 0.15)$ & \\
\hline$v=4$ & $(-67.259 \pm 0.005)$ & $(+0.52 \pm 0.20)$ & $(+10.212 \pm 0.18)$ & & \\
\hline \multicolumn{6}{|c|}{ Previous results } \\
\hline \multicolumn{6}{|c|}{ Hughes and Grabner" } \\
\hline$v=0$ & $(-70.31 \pm 0.10)$ & \multicolumn{4}{|c|}{$(11 \pm 3)$ (magnitude only) } \\
\hline$v=1$ & $(-69.54 \pm 0.10)$ & \multicolumn{4}{|c|}{$(13 \pm 3)$ (magnitude only) } \\
\hline$v=2$ & $(-68.71 \pm 0.10)$ & \multicolumn{4}{|c|}{$(10 \pm 3)$ (magnitude only) } \\
\hline$v=3$ & $(-67.99 \pm 0.10)$ & \multicolumn{4}{|c|}{$(10 \pm 3)$ (magnitude only) } \\
\hline$v=4$ & $(-67.20 \pm 0.10)$ & \multicolumn{4}{|c|}{$(14 \pm 4)$ (magnitude only) } \\
\hline \multicolumn{6}{|c|}{ Lew et $a b^{\mathrm{b}}$} \\
\hline$v=0$ & $(-70.340 \pm 0.030)$ & & & & \\
\hline \multicolumn{6}{|c|}{ Bonczyk and Hughes ${ }^{\mathbf{c}}$} \\
\hline$v=0$ & $(-70.3405 \pm 0.0004)$ & $(+0.525 \pm 0.01)$ & $(+10.53 \pm 0.07)$ & $(+0.93 \pm 0.05)$ & $(+0.23 \pm 0.06)$ \\
\hline \multicolumn{6}{|c|}{ Gräff, Schönwasser, and Tonutti ${ }^{\mathrm{d}}$} \\
\hline$v=0$ & $(-70.3410 \pm 0.0026)$ & $(+0.479 \pm 0.048)$ & $(+10.4 \pm 0.7)$ & $(+0.69 \pm 0.22)$ & $(+0.36 \pm 0.23)$ \\
\hline$v=1$ & $(-69.556 \pm 0.011)$ & $(+0.52 \pm 0.20)$ & $(+10.6 \pm 0.4)$ & $(+0.81 \pm 0.23)$ & $(+1.0 \pm 1.3)$ \\
\hline
\end{tabular}

See Ref. 3. 'b See Ref. 4. ' See Ref. 9. ' See Ref. 13.

the coefficients in the expansion

$$
q(R)=g^{(0)}+q^{(1)} R+q^{(2)} R^{2}+\cdots
$$

can be derived from the spectrum. [Equation (5) above might be called an "electric gradient function" in analogy to the powers-of- $R$ series expansion for the electric dipole moment which is called the "dipole moment function".] The internuclear separation is also $J$ and $v$ dependent, of course, so relations between the coefficients in the expansions of Eqs. (4) and (5) above can be derived. This has been done $e^{5,26}$ in approximations which predict a variation of $q$ which is linear in $v$ and in $[J(J+1)]$. However the coefficient $q^{(2 v)}$ is about $5 \mathrm{kHz}$, while the values for $e Q q^{(J)}$ are less than $50 \mathrm{~Hz}$, so it seems likely that utilization of the $q^{(2 v)}$ terms, as measured in a variety of molecules, may aid in deriving more reliable values of the coefficients in the electric gradient function.

The quoted values for $c_{2}$ (as well as for the rest of the hfs constants) in each vibrational state are the result of a fit to all the observed lines of that state; however, it happens that the difference between the frequencies of the $\left(\frac{3}{2}, 2\right)-\left(\frac{7}{2}, 4\right)$ and $\left(\frac{3}{2}, 1\right)-\left(\frac{7}{2}, 3\right)$ transitions is itself an excellent measure of $c_{2}$. This frequency dif-

${ }^{26}$ A. G. Makhanek, Opt. Spectry. 9, 214 (1960); 11, 6 (1961) [English transl.: Opt. i Spectroskopiya 9, 412 (1960); 11, 12 (1961) ]; H. J. Zeiger and D. I. Bolef, Phys. Rev. 85, 788 (1952). ference, or splitting, is denoted by $S$; we can express it as

$$
S=1.943 c_{2}+0.023 c_{3}+0.057 c_{4},
$$

where $S$ and the constants $c_{2}, c_{3}$, and $c_{4}$ are in units of kilohertz. We know that $c_{3}$ is about $0.8 \mathrm{kHz}$, and $c_{4}$ is less than $0.2 \mathrm{kHz}$ for the lower vibrational states, so unless $c_{3}$ or $c_{4}$ become much larger as $v$ increases (which would be most surprising), we can write $S=1.943 c_{2}$ to an accuracy of $0.05 \mathrm{kHz}$ or better. Assuming that $c_{3}$ and $c_{4}$ do not change appreciably, we can compute $c_{2}$ directly from the splitting $S$ and compare it to the least-squares value in the various vibrational states; this is shown in Table III. The discrepancy between

TABLE III. Values of spin-rotation interaction $c_{2}$.

\begin{tabular}{cccc}
\hline & $c_{2}\left(\begin{array}{c}\text { from } S) \\
(\mathrm{kHz})\end{array}\right.$ & $\begin{array}{c}c_{2}(\text { from least squares }) \\
(\mathrm{kHz})\end{array}$ & $n_{v}{ }^{\mathbf{8}}$ \\
\hline 0 & 10.55 & 10.615 & 10 \\
1 & 10.46 & 10.483 & 6 \\
2 & 10.42 & 10.406 & 6 \\
3 & 10.21 & 10.205 & 5 \\
4 & 10.21 & 10.212 & 5
\end{tabular}

a The number of lines observed for each vibrational state is given in the column labeled $n_{v}$. For $v=3$ and 4 , not all of the lines of the $F_{1}=\frac{3}{2}$ to $F_{t}=\frac{5}{2}$ transitions were resolved. See Table I. 
the $v=0$ values arises because the least-squares value of $c_{2}$ has been adjusted along with the rest of the hfs constants to match a set of 10 lines; the resultant value of $c_{2}$ is thus a compromise which yields a predicted value of $S$ that is $0.15 \mathrm{kHz}$ larger than the experimental value. The least-squares values of $c_{2}$ for the higher vibrational states, however, are less of a compromise because fewer lines are matched, and we find the values of $S$ for $v>0$ calculated from these leastsquares values to be within $0.05 \mathrm{kHz}$ of the measured splittings.

The discrepancy between the values for $c_{2}$ in $v=0$ probably has its origin in the systematic errors which can arise in choosing line centers. For example the splitting between the $\left(\frac{3}{2}, 1\right)-\left(\frac{5}{2}, 3\right)$ and $\left(\frac{3}{2}, 1\right)-$ $\left(\frac{5}{2}, 2\right)$ transition frequencies should be identical to that seen between the $\left(\frac{3}{2}, 2\right)-\left(\frac{5}{2}, 3\right)$ and $\left(\frac{3}{2}, 2\right)-\left(\frac{5}{2}, 2\right)$ lines, however these splittings appear to be 2.0 and 1.6 $\mathrm{kHz}$, respectively, for all the vibrational levels in which these lines are resolved $(v=0,1,2$ and part of $v=3)$. The line patterns of the different vibrational states are remarkably similar and the line assignments seem unambiguous, so we can only conclude that reliance on the apparent centers of individual, very-weak-field spectral lines to better than $200 \mathrm{~Hz}$ may not be without its dangers. In spite of this difficulty, the similarity of line shapes observed in the different vibrational states appears to permit a useful measurement of the change in relative value of the constants $e q_{1} Q_{1}$ and $c_{2}$ with vibrational state. The changes in quadrupole coupling are reasonable and in accord with the findings in other molecules, ${ }^{27}$ but it can be remarked that the apparent equality of $c_{2}(v=3)$ and $c_{2}(v=4)$ is unexpected in view of the progressive decrease of $c_{2}$ in the states $v=0,1$, and 2.

For consistency we quote the least-squares values for the final results of all hfs constants given in Table II. Although the central values thus obtained may not coincide with values for the constants obtained in other analyses of the same data, as we have seen for $c_{2}$ in the $v=0$ state, the quoted uncertainty in the final values encompasses these other values as well.

We believe that noise is not the limitation to pre-

${ }_{27}$ A. J. Hebert, F. W. Breivogel, and K. Street, J. Chem. Phys. 41, 2368 (1964) ; C. D. Hollowell, A. J. Hebert, and K. Street, ibid. 41, 3540 (1964). cision with the present experiment; rather it is the Stark effect from the weak dc and rf electric fields that makes it hard to specify the zero-field line centers to better accuracy than quoted here. These residual Stark effects seem to be the most serious obstacle to a more accurate measurement of the molecular constants. It is true that a very quiet signal would permit an extensive study of the line shapes, but the very-weak-field spectroscopy reported here is severely hampered by the presence of three or more unresolved components, separated from each other by $100 \mathrm{~Hz}$ or so, which go into each observed spectral line.

MBER spectroscopy has been done at high electric fields of several hundred volts per $\mathrm{cm}$, where the line components are well separated, but problems can arise in that the Stark energy levels are dependent on the square of the total (effective sum of $\mathrm{dc}$ and rf) electric field $\varepsilon$. Since the linewidth $\delta f$ depends on $2 \varepsilon \delta \varepsilon$, inhomogeneities in the if electric field may prove to be the limiting factor. Major progress with very-weak-field spectroscopy appears to depend on resolving the individual $F, M_{F}$ components of the transitions at field strengths which are low enough so that field inhomogeneity is not a problem and low enough so calculations do not become unwieldy. An apparatus with an intrinsic linewidth of $100 \mathrm{~Hz}$ or less, with a dc field of a few volts per centimeter, and with a residual magnetic field of less than $0.01 \mathrm{G}$ is probably necessary.

\section{ACKNOWLEDGMENTS}

We would like to thank Professor P. A. Franken and Professor R. H. Sands along with many other members of the Resonance Group for advice and support. We have enjoyed the cheerful cooperation of Mr. Hermann Roemer and Mr. August Wagner and their staff of instrument makers in the University of Michigan Physics shop.

One of us (J.C.Z.) would like to acknowledge the invaluable experience acquired while working in the Yale molecular beam laboratory of Professor Vernon Hughes. The early data on the $v=0{ }^{86} \mathrm{Rb}^{19} \mathrm{~F}$ spectrum, obtained in experiments done together with George Chamberlain and Paul Bonczyk, greatly facilitated the study of the excited vibrational states. 